\title{
Value of thromboelastography combined with anticoagulant detection in patients over 60 years of age with cardiovascular or cerebrovascular disease
}

\author{
Fenglian Zhao, Pengyue Yu, Xiaoyan Lv, Mengyu Pang, Lijuan Zhao, Xiaohua Zhang, Liyan Zhao \\ Department of Clinical Laboratory, the Second Hospital of Jilin University, Changchun, China \\ Contributions: (I) Conception and design: L Zhao; (II) Administrative support: X Lv; (III) Provision of study materials or patients: M Pang, L Zhao; (IV) \\ Collection and assembly of data: X Zhang; (V) Data analysis and interpretation: F Zhao; (VI) Manuscript writing: All authors; (VII) Final approval of \\ manuscript: All authors. \\ Correspondence to: Liyan Zhao. Department of Clinical Laboratory, the Second Hospital of Jilin University, Changchun, China. \\ Email: Zhaoliy@jlu.edu.cn.
}

Background: Cardiovascular disease (CVD) and cerebrovascular disease (CBVD) are common diseases in
middle-aged and elderly people with high morbidity, mortality and disability rates. Individualized assessment
and treatment are helpful to improve the quality of life and prognosis of patients. Thrombelastography (TEG)
is a method to evaluate the coagulation function of patient with whole blood. In this study, we investigated
the correlation between TEG indicators and traditional indicators of coagulation, and evaluated the
diagnostic value of TEG combined with indicators of coagulation in elderly patients with CVD and CBVD.
Methods: We retrospectively analyzed the thrombelastographic indices and traditional indicators of
coagulation in 285 cases of elderly patients with CBVD or CVD. All measures were performed by the
Department of Clinical Laboratory in the Second Hospital of Jilin University between January 2016 and
December 2018 .

Results: Compared with the control group, the platelet count, mean platelet volume (MPV), and APTT were increased in the CBVD group. $\mathrm{K}$ value, MPV, and the MPV/PLT ratio were higher, but fibrinogen levels and the PT were lower in the CVD group than in the control group. In the CVD and CBVD groups, the $\mathrm{R}$ value was positively correlated with APTT, the $\mathrm{K}$ value was negatively correlated with fibrinogen, $\alpha$ angle was positively correlated with fibrinogen, and the MA was positively correlated with the platelet count and fibrinogen, but negatively correlated with MPV/PLT. The FDP was significantly higher, whereas the D-dimer/FDP ratio was lower in the CBVD group than in the CVD group.

Conclusions: The combined detection of TEG and traditional indicators is more effective than detection using only routine indices in the assessment of the coagulation status of elderly patients with CVD or CBVD. The MPV/PLT and D-dimer/FDP ratios can be used as indices of anticoagulant function.

Keywords: Thromboelastography; cerebrovascular disease (CBVD); cardiovascular disease (CVD); coagulation function; mean platelet volume

Submitted Mar 13, 2021. Accepted for publication Apr 28, 2021.

doi: 10.21037/apm-21-822

View this article at: http://dx.doi.org/10.21037/apm-21-822

\section{Introduction}

The aging of our society and dietary changes have contributed to the high incidence of adverse cardiovascular and cerebrovascular events, which threaten the lives and health of middle-aged and elderly people. There are reportedly about 290 million patients with cardiovascular disease (CVD) or cerebrovascular disease (CBVD) in China, accounting for $42.5 \%$ and $44.6 \%$ of the causes of death in 
urban and rural residents, respectively (1). CVD and CBVD also reduce an individual's life expectancy by more than 2 years per year. Moreover, CVD and CBVD (especially ischemic stroke) are the main causes of disability and death in Chinese adults (2-8). These diseases are usually treated with antiplatelet and anticoagulant therapies, including clopidogrel and aspirin, which are widely used for the treatment of CBVD in elderly patients. However, certain polymorphisms, such as those of metabolic enzymes and drug receptors, and some other individual differences can affect drug responsiveness, meaning that not all patients show the same outcome despite identical treatment plans. Therefore, individualized treatment is necessary to improve the outcomes of patients with CVD or CBVD.

Standardized and effective monitoring is essential for individualized treatment. The development of thromboelastography (TEG) has compensated for the deficiencies of traditional methods for measuring coagulation function. TEG provides a comprehensive assessment of blood coagulation. It has been used to detect coagulation function and evaluate disease in patients with severe trauma, surgery, or liver transplantation. TEG also plays a vital role in the decision-making process regarding clinical anticoagulation and hemostasis $(9,10)$. Many studies have used TEG to evaluate the coagulation status of patients with different types of arteriovenous thrombosis and to predict deep venous thrombosis (DVT) and CBVD. In clinical practice, we primarily monitor antiplatelet and anticoagulation treatments with TEG and routine anticoagulation tests. However, single clinical indicators cannot effectively monitor treatment outcomes. Therefore, TEG could improve the diagnosis rates of diseases when combined with other relevant laboratory tests (11-13).

To our knowledge, no reports have investigated the use of TEG combined with routine coagulation tests for the assessment of CVD or CBVD in older patients. Therefore, the purpose of this research was to explore the relationship between TEG and traditional indicators of coagulation, and to assess the diagnostic value of TEG combined with indicators of coagulation in elderly patients with CVD or CBVD.

We present the study in accordance with the STROBE reporting checklist (available at http://dx.doi.org/10.21037/ apm-21-822).

\section{Methods}

\section{Data collection and classification}

We retrospectively analyzed 113 elderly patients diagnosed with CBVD and 172 elderly patients diagnosed with CVD who were admitted to the Second Hospital of Jilin University (Changchun city, Jilin province, China) between January 2016 and December 2018. A total of 83 sex- and age-matched physical examination participants were selected as the control group during the same period. The results of TEG, a routine blood coagulation test, and platelet (PLT) counts were recorded, and are shown in Table 1. All the enrolled participants were diagnosed with CVD or CBVD and had not received anticoagulant or antiplatelet drugs within the 3 months preceding admission. This study conformed to the tenets of the Declaration of Helsinki (as revised in 2013) and was approved by the Institutional Ethics Committee of the Second Hospital of Jilin University (2019-063). Because of the retrospective nature of the study, the requirement for informed consent was waived.

\section{TEG}

Peripheral blood samples, anticoagulated with sodium citrate, were obtained from all the participants before the treatment. TEG was performed with routine laboratory methods using the $\mathrm{TEG}^{\circledR} 5000$ Thrombelastograph $^{\circledR}$ Hemostasis Analyzer and its supporting kit (Haemoscope Inc., Beaumont, Texas, USA) in accordance with the manufacturer's protocol. The relevant test indicators included the coagulation reaction time ( $\mathrm{R}$ value), clot formation time ( $\mathrm{K}$ value), clot formation rate ( $\alpha$ angle), and maximum clot intensity (MA value).

\section{Routine blood coagulation tests}

The prothrombin time (PT), fibrinogen, and activated partial thromboplastin time (APTT) were measured using the ACL Top 700 detection system and supporting kit (Werfen, Barcelona, Spain). The levels of D-dimer and fibrin/fibrinogen degradation products (FDP) were measured using the Coapresta ${ }^{\circledR} 2000$ detection system and supporting kit (Sekisui, Tokyo, Japan).

\section{Platelet assay}

Peripheral venous blood samples, anticoagulated with ethylenediaminetetraacetic acid, were measured within $8 \mathrm{~h}$ of sample collection. The PLT count and average PLT volume (MPV) were determined using the Sysmex XN2000 Hematology Analyzer and its supporting reagents (Sysmex, Kobe, Japan). 
Table 1 TEG, coagulation function, and platelet test results in different groups

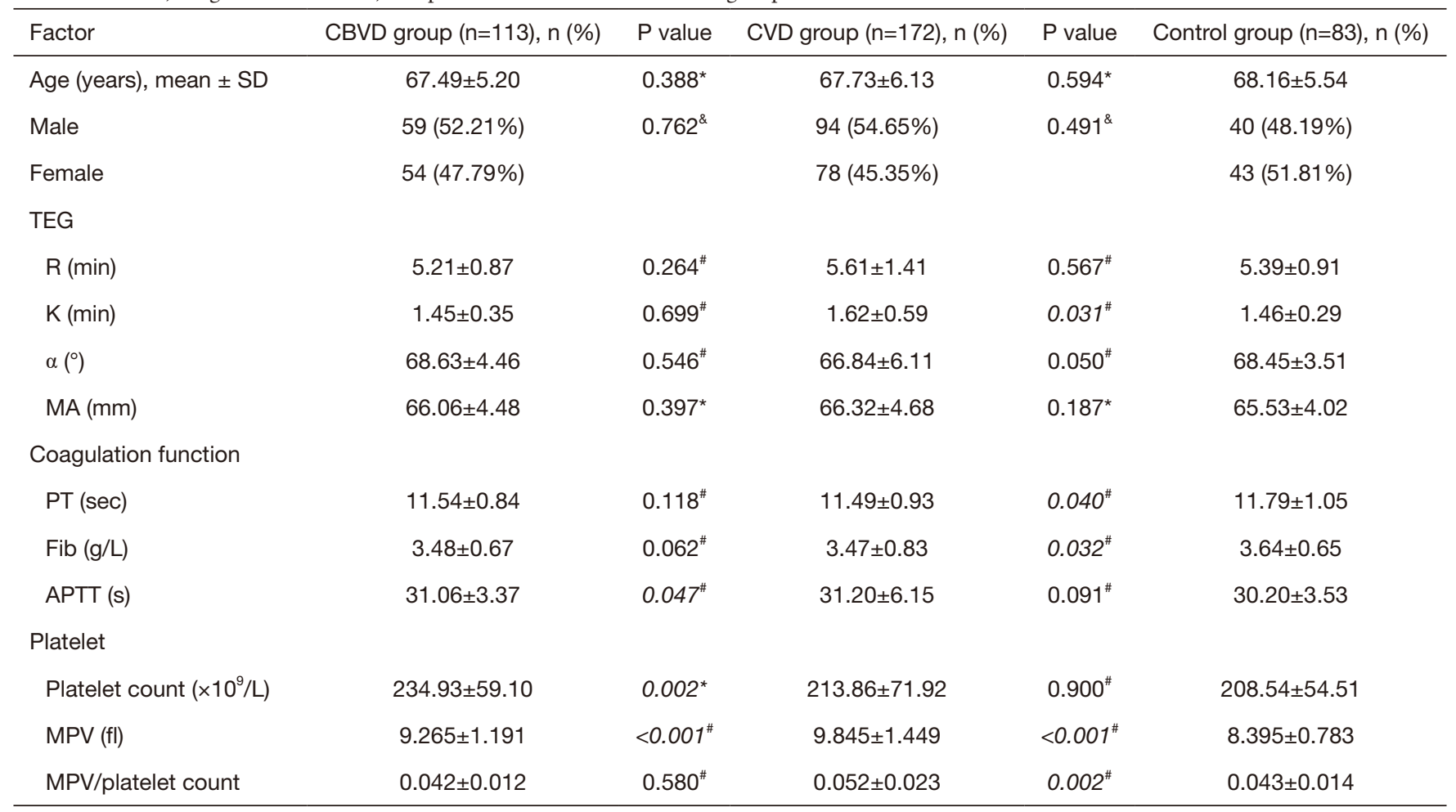

*, $t$-test; ${ }^{\&}, \chi^{2}$ test; ", Mann-Whitney test. Italic values indicate statistical significance at $\mathrm{P}<0.05$ or $\mathrm{P}<0.01$. TEG, thromboelastography; $\mathrm{SD}$, standard deviation; $\mathrm{R}$, coagulation reaction time; K, clot formation time; $\alpha$ angle, clot formation rate; MA, maximum clot intensity; PT, prothrombin time; Fib, fibrinogen; APTT, activated partial thromboplastin time; MPV, mean platelet value.

\section{Statistical analysis}

All data were analyzed with SPSS 24.0 (SPSS Inc., Chicago, IL, USA). Continuous variables (measurement data) with a normal distribution are expressed as the mean \pm standard deviation (SD), while abnormally distributed variables are expressed as the median (25th-75th percentile). The differences between the two groups were analyzed using the $\mathrm{t}$ test or Mann-Whitney test. $\mathrm{P}<0.05$ was considered to demonstrate statistically significant differences. The correlation analysis of parametric variables was performed using Pearson's correlation coefficient, while the correlation analysis of nonparametric variables was performed using Spearman's correlation coefficient.

\section{Results}

\section{Correlation of the $R$ value with PT and APTT}

The $R$ value is an indicator of the coagulation reaction time and reflects the activity of coagulation factors. Therefore, we analyzed the correlations between the $R$ value and the traditional indicators $\mathrm{PT}$ and APTT. There were no obvious differences in the $\mathrm{R}$ value $(\mathrm{P}=0.264)$ and $\mathrm{PT}$ $(\mathrm{P}=0.118)$ between the CBVD group and the control group, but APTT was increased in the CBVD group $(\mathrm{P}=0.047)$. The $\mathrm{R}$ value was positively correlated with APTT in the CBVD group $(\mathrm{r}=0.295, \mathrm{P}=0.002)$. In the CVD group, $\mathrm{PT}$ was obviously lower than in the control group $(\mathrm{P}=0.040)$ and the $\mathrm{R}$ value was positively correlated with APTT $(\mathrm{r}=0.176, \mathrm{P}=0.021)$, as shown in Table 1, Figure $1 A$ and $B$.

\section{Correlations of the a angle and $K$ value with fibrinogen}

Because the $\alpha$ angle and $\mathrm{K}$ value are indicators of fibrinogen activity, we also determined whether these indicates were correlated with fibrinogen. There were no obvious statistical differences in the 3 indicators between the CBVD group and the control group. In the CBVD group, the K value was negatively correlated with fibrinogen $(r=-0.293$, $\mathrm{P}=0.002)$, whereas the $\alpha$ angle was positively correlated with fibrinogen $(r=0.345, \mathrm{P}<0.001)$. In the $\mathrm{CVD}$ group, the $\mathrm{K}$ value was higher $(\mathrm{P}=0.031)$ and the fibrinogen value 

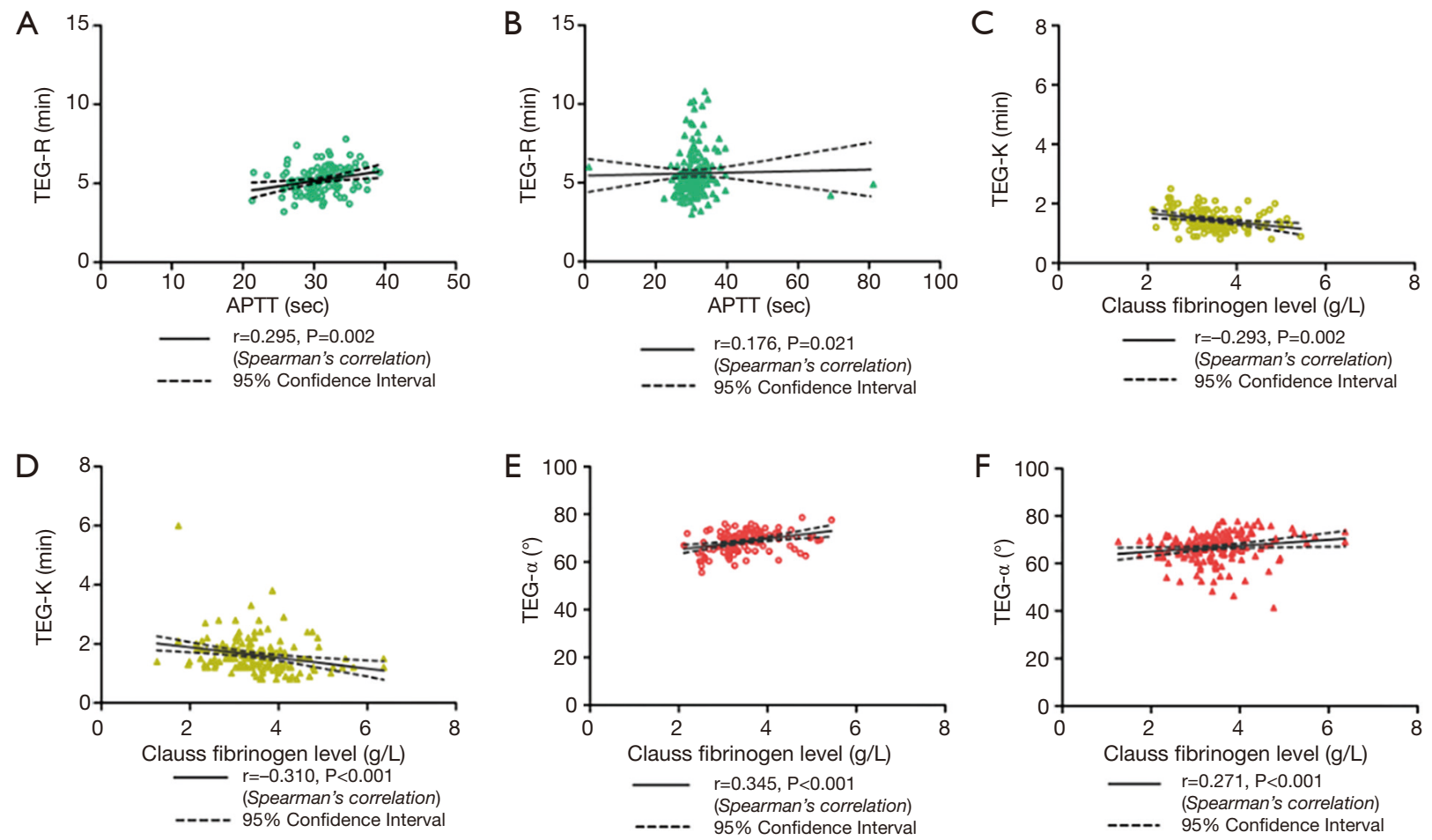

Figure 1 Correlation analysis of TEG indices with activated partial thromboplastin time (APTT) and Clauss fibrinogen level. (A) Correlation between APTT and the R value in the cerebrovascular disease group; (B) correlation between APTT and the R value in the cardiovascular disease group; (C) correlation between the Clauss fibrinogen level and the $\mathrm{K}$ value in the cerebrovascular disease group; (D) correlation between the Clauss fibrinogen level and the $\mathrm{K}$ value in the cardiovascular disease group; (E) correlation between the Clauss fibrinogen level and the $\alpha$ angle in the cerebrovascular disease group; (F) correlation between the Clauss fibrinogen level and the $\alpha$ angle in the cardiovascular disease group.

was lower $(\mathrm{P}=0.032)$ compared with the control group. Furthermore, the $\mathrm{K}$ value showed a negative correlation with fibrinogen $(\mathrm{r}=-0.310, \mathrm{P}<0.001)$ whereas the $\alpha$ angle showed a positive correlation with fibrinogen $(\mathrm{r}=0.271$, $\mathrm{P}<0.001$ ), as shown in Table 1 and Figure $1 C, D, E, F$.

\section{Correlations of MA with PLT count, MPV, and MPV/PLT}

To investigate the relationship between MA and PLT function, we determined whether MA was correlated with the PLT count, MPV, and MPV/PLT. There was no obvious difference in MA between the CBVD group and the control group. The PLT count and MPV of the CBVD group were increased compared to the control group, but MPV/PLT did not differ between the two groups. MA showed a positive correlation with the PLT count $(\mathrm{r}=0.540$, $\mathrm{P}<0.001)$ and fibrinogen $(\mathrm{r}=0.417, \mathrm{P}<0.001)$, but a negative correlation with MPV/PLT $(r=-0.444, \mathrm{P}<0.001)$ in the
CBVD group. As compared with the control group, MPV and MPV/PLT were increased in the CVD group, but neither MA nor PLT differed obviously between the two groups. In the CVD group, MA showed a highly positive correlation with PLT $(r=0.420, \mathrm{P}<0.001)$ and fibrinogen $(\mathrm{r}=0.418, \mathrm{P}<0.001)$, and showed a negative correlation with MPV/PLT $(\mathrm{r}=-0.295, \mathrm{P}<0.001)$, as shown in Table 1 and Figure 2, A,B,C,D,E,F.

\section{Distribution characteristics of D-dimer and FDP in the CVD and CBVD groups}

The distributions of D-dimer and FDP in the CBVD and CVD groups were analyzed and the results are shown in Table 2 and Figure 3. D-dimer was not obviously different between the CVD and CBVD groups $(\mathrm{P}=0.164)$. However, the $\mathrm{D}$-dimer/FDP ratio $(\mathrm{P}=0.001)$ and $\mathrm{FDP}(\mathrm{P}<0.001)$ were markedly different between these two groups. 

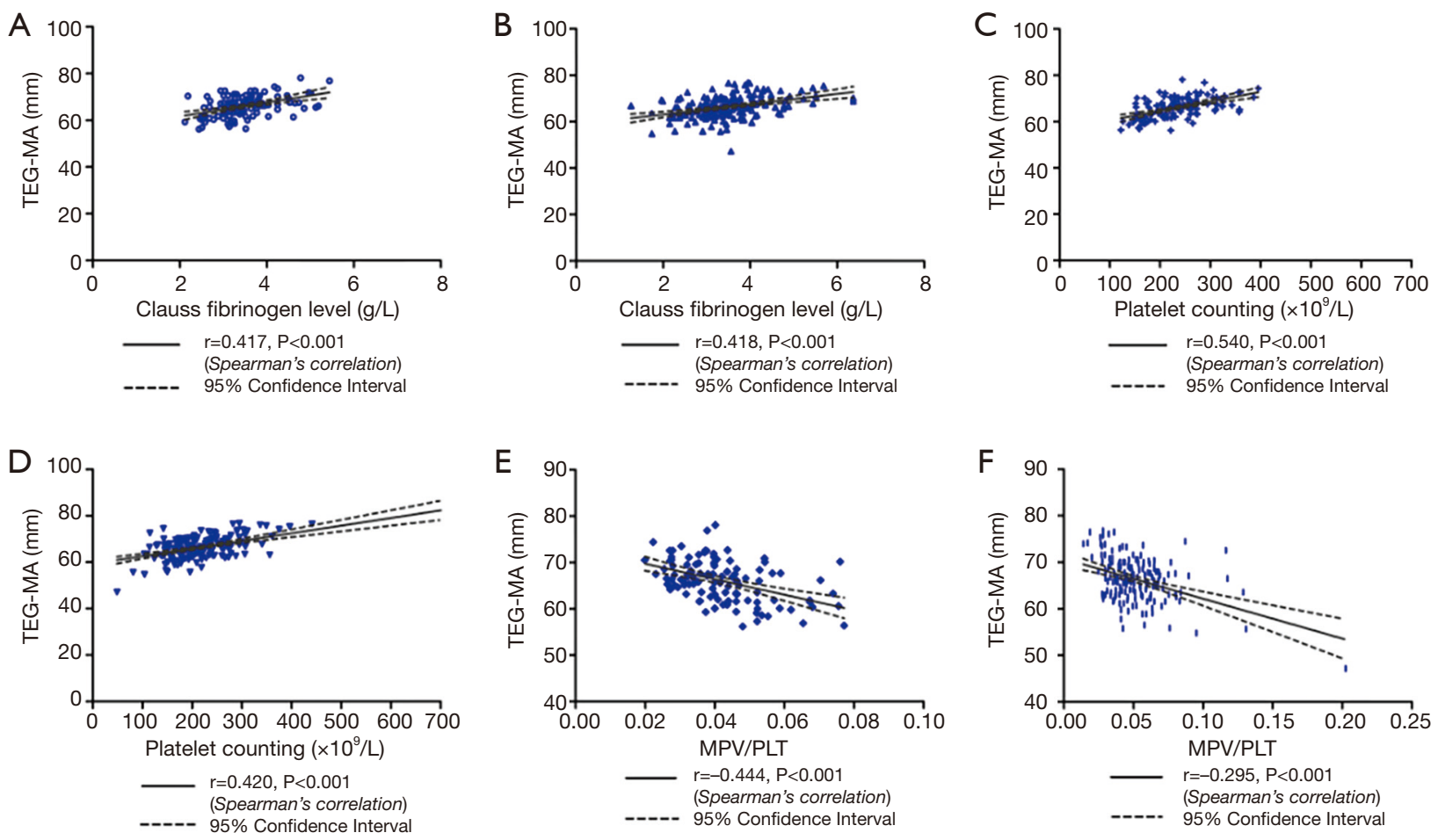

Figure 2 Correlation analysis of maximum clot intensity (MA) with the Clauss fibrinogen level, platelet (PLT) count, and the mean PLT volume (MPV)/PLT ratio. (A,C,E) Correlations between MA and the Clauss fibrinogen level (A), PLT level (C), and MPV/PLT (E) in the cerebrovascular disease group. (B,D,F) Correlations between MA and the Clauss fibrinogen level (B), PLT level (D), and MPV/PLT (F) in the cardiovascular disease group.

\section{Discussion}

CVD and CBVD are the main pathologies threatening human health and are major causes of death (14). Inflammation and endothelial cell activation and dysfunction, especially abnormal PLT activation, are the main pathogeneses of CVD and CBVD (15). Chronic inflammation can also lead to premature activation of PLT and promotes the activation of thrombin, fibrinogen, and other coagulation factors to form pathological clots (16). In this study, we found that APTT was increased in the CBVD group, but there were no obvious differences in PT or fibrinogen, which are two established indices of blood clotting function. However, compared with the control group, PT and fibrinogen were decreased in the CVD group, whereas no obvious difference was detected in APTT. These results indicate that the CVD patients tended to be hypercoagulant, and showed greater fibrinogen consumption in the process of blood vessel damage and PLT activation. Being an acute-phase reactant, fibrinogen does not increase until at least $6 \mathrm{~h}$ after tissue injury. After
6-12 h, it increases to different degrees as the tissue damage and repair processes progress. Studies have also shown that the fibrinogen/albumin ratio is significantly increased in sufferers with stable coronary heart disease or slow coronary artery flow (15), and can be used to predict the prognosis of these diseases.

Unlike traditional methods to assess coagulation, TEG requires whole blood samples to measure the complete clotting process via the kaolin-activated thrombin pathway in vitro. TEG can be used to quantify the formation and strength of blood clots in vitro, and is therefore a sophisticated method for the analysis of clotting and fibrinogen function (17). TEG is also widely used to evaluate and monitor various CVDs and CBVDs. The $\mathrm{R}$ value generally decreases within $6 \mathrm{~h}$ of the onset of cerebral hemorrhage, whereas the MA value usually increases $36 \mathrm{~h}$ after its onset (18). In this study, there were no marked differences in the $\mathrm{R}, \mathrm{K}, \alpha$ angle, or MA value between the $\mathrm{CVD}$ and control groups. However, the $\mathrm{K}$ values were higher in the CVD group than those in the control group 
Table 2 D-dimer and FDP levels of patients with CBVD or CVD

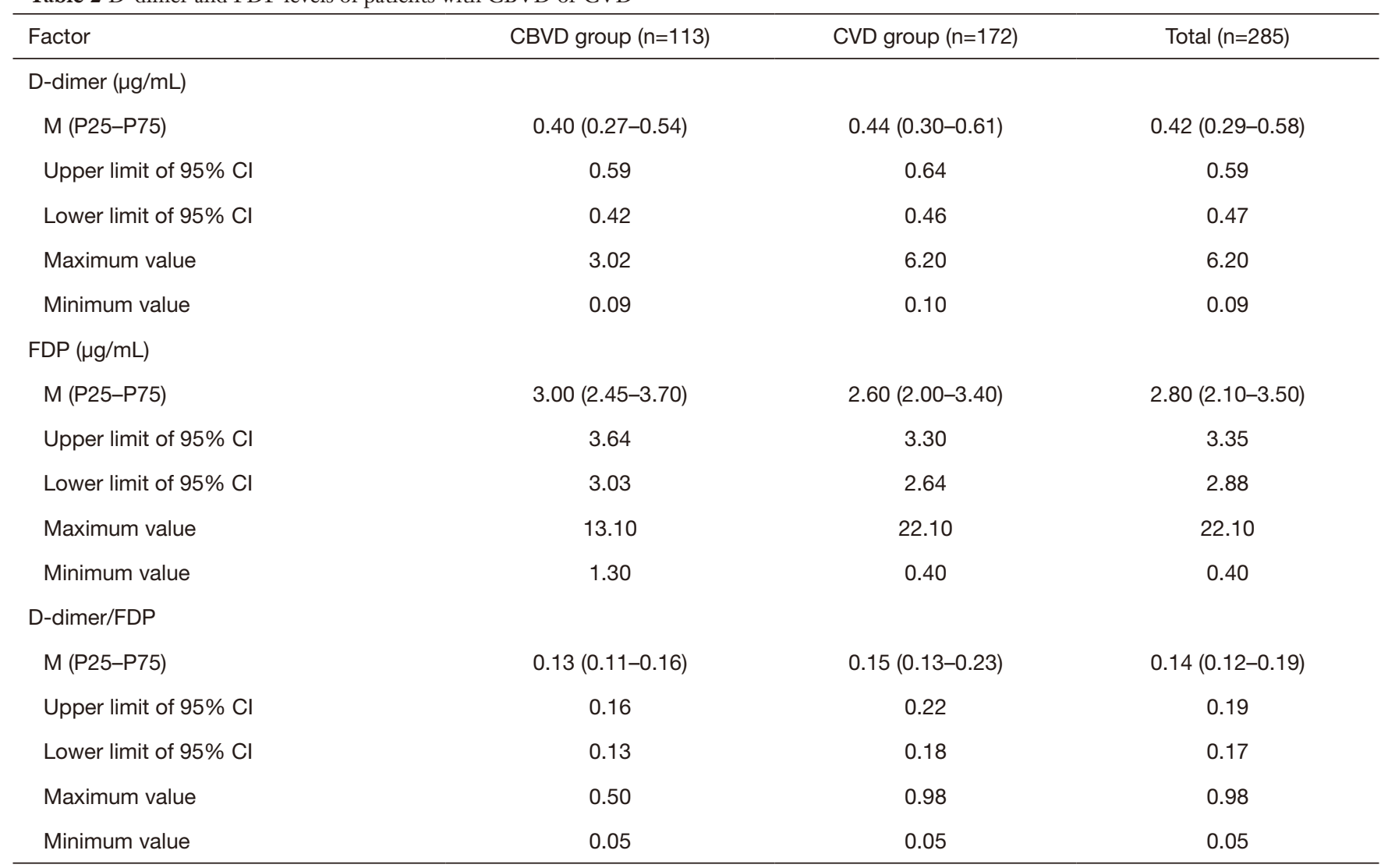

FDP, fibrin/fibrinogen degradation products; CBVD, cerebrovascular disease; CVD, cardiovascular disease; M, median; P25, $25^{\text {th }}$ percentile; $\mathrm{P} 75,75^{\text {th }}$ percentile; $\mathrm{Cl}$, confidence interval.

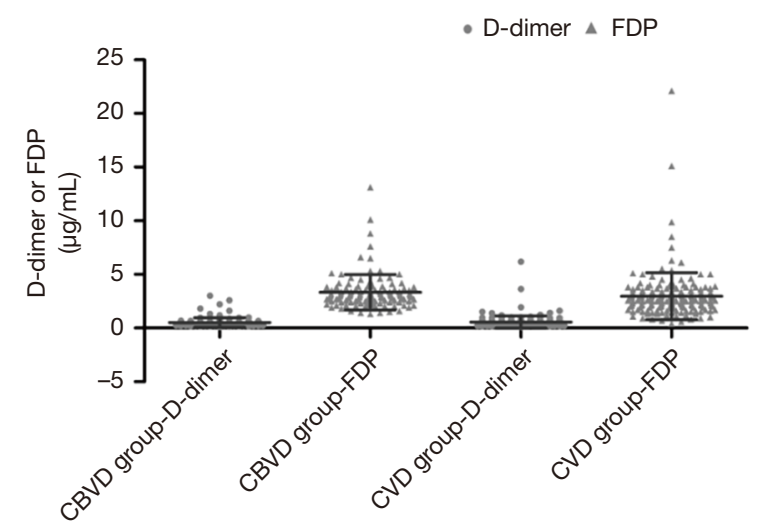

Figure 3 Distributions of the levels of D-dimer and fibrin/ fibrinogen degradation products (FDP) in patients with cerebrovascular disease or cardiovascular disease. as the $\mathrm{K}$ value is an index of fibrinogen function. These results support our hypothesis that fibrinogen consumption is increased in the early stages of CVD. The differences in the MA values and $\alpha$ angles between the CVD and control groups in this study may be attributable to the coagulation status of the elderly.

Regardless of how these methods evolve over time, they always focus on the pathogenesis of vascular diseases and the basic theory of coagulation. Therefore, we assessed whether the TEG indices are correlated with the traditional indicators of coagulation. First, the $\mathrm{R}$ value is the coagulation reaction time, an indicator of coagulation factor activity. Because the activator tested by TEG is kaolin, the $\mathrm{R}$ value mainly reflects the activity of coagulation factors in endogenous pathways. We found that the $R$ value was 
positively correlated with APTT in both CBVD and CVD. Therefore, changes in the $\mathrm{R}$ value only represent an increase or decrease in the activity of endogenous coagulation factors. However, it has also been reported that the $\mathrm{R}$ value can be used to monitor low-molecular-weight heparin. After enoxaparin was administered, the $\mathrm{R}$ values for the heparinase-coated cup were shorter than those for the standard cup by more than $1 \mathrm{~min}$, which suggests that the risk of developing venous thromboembolism (VTE) was reduced by enoxaparin (19). The $\mathrm{R}$ value can also be used to monitor newer anticoagulants, such as rivaroxaban (20). Another study showed that in 10 stroke patients given rivaroxaban, the $\mathrm{R}$ value increased from $5.8 \pm 0.5$ at baseline to $11.4 \pm 1.0$ at $2 \mathrm{~h}$, and increased further (21). An $\mathrm{R}$ value below $3.45 \mathrm{~min}$ is a good predictor of early neurological deterioration in acute ischemic stroke patients, with a sensitivity of $87.9 \%$ and a specificity of $40.3 \%$ (22).

Fibrinogen, which is reflected by the $\mathrm{K}$ value and $\alpha$ angle, is an important coagulation factor that forms stable cross-linked fibrinogen under the action of Factor XIII. Fibrinogen activation can mediate PLT aggregation through the membrane glycoprotein GPIIb/IIIa receptor on the PLT surface, acting as a bridge to stabilize blood clots (23). In TEG, the blood clot strength is affected by both fibrinogen and Factor XIIIa (24). An increased K value and a decreased $\alpha$ angle indicate reduced fibrinogen function. This study also confirmed that the $\mathrm{K}$ values of elderly CBVD and CVD patients correlated negatively with fibrinogen, whereas the $\alpha$ values of the two groups correlated positively with fibrinogen.

The MA value is the maximum clot strength and reflects the combined effect of PLT and fibrinogen, to which PLT contributes $80 \%$ and fibrinogen only $20 \%$. Therefore, MA is mainly used to evaluate PLT function (25). An increase in MA indicates that PLT function is enhanced and the risk of thrombosis is correspondingly increased. However, a cohort study of 240 patients who underwent non-cardiac surgery found that the incidence of DVT complications in patients with MA $>68 \mathrm{~mm}$ was higher than in patients with MA $<68 \mathrm{~mm}$ (8.4\% and $1.4 \%$, respectively), and that the MA values for all patients with myocardial infarction in the study were $>68 \mathrm{~mm}$ (26). The increase in MA has also been shown to be closely related to the ischemia time after coronary artery stenting (27-29). The mortality of patients with significantly increased MA was increased by 3.8 -fold after stenting (29). This highlights the role of PLT in the formation of blood clots.

However, several studies have shown that PLT function is related to PLT volume and PLT count. Increased MPV indicates an increased PLT volume, more dense particles, high enzyme activity, enhanced adhesion and aggregation functions, and greater susceptibility to thrombosis (30-32). Other studies have confirmed that MPV is higher in patients with a known DVT and diabetes mellitus with myocardial infarct than in healthy subjects $(33,34)$. We also found that the PLT count and MPV were dramatically increased in the CBVD group and that MPV and the MPV/ PLT ratio were clearly increased in the CVD group, which indicates that PLT function is enhanced in the diseased state. Therefore, MPV and the MPV/PLT ratio can be used as evaluation indices for CVD and CBVD.

We also analyzed the correlations between the MA value, PLT, and fibrinogen to evaluate the function of fibrinogen in blood clot formation and dissolution. We confirmed that the MA value correlated positively with the PLT count and fibrinogen in CVD and CBVD, which suggests that MA is still mainly dependent on the PLT count. However, the contribution of fibrinogen to MA may be more than $20 \%$. Therefore, anticoagulation therapy as well as antiplatelet therapy should be monitored during clinical treatment.

Additionally, we analyzed the levels of FDP and D-dimer in elderly patients with CVD or CBVD to further evaluate their thrombogenic status. There was no obvious difference in the D-dimer levels between the two disease groups, whereas there was a dramatic difference in the FDP levels and the D-dimer/FDP ratio. Compared with the CVD group, the FDP level was increased in the CBVD group. In recent years, many clinical studies have confirmed that the joint detection of D-dimer and FDP can be used to assess all kinds of embolism risks and to monitor the treatment of CBVD and CVD (31), especially in patients with acute ischemic stroke (AIS) or transient ischemic attack (TIA) complicated with type A aortic dissection (35). D-dimer can be used as an effective biomarker for the monitoring and treatment of patients with stroke suspected to be derived from VTE, whereas a normal D-dimer level can be used to exclude VTE in patients whose stroke is not derived from VTE (36). Some researchers also believe that the D-dimer level at the onset of stroke is positively correlated with the risk stratification of stroke, and is an independent risk factor for ischemic stroke (37). Because D-dimer level increases with age, the cut-off value for the elderly population must be adjusted according to the patient's age (38). This suggests that the combined detection of D-dimer and FDP may be more valuable than single $\mathrm{D}$-dimer in the diagnosis of disease. 
In conclusion, TEG provides a more comprehensive assessment of the coagulation status of elderly patients with CVD or CBVD than traditional indices, and is a powerful complement to traditional coagulation function tests. The MPV/PLT and D-dimer/FDP ratios can also be used as comprehensive indicators of the blood coagulation status of the patient.

\section{Acknowledgments}

Funding: This research was funded by Training Program of National Natural Science Foundation of China (Grant No. 2019SRCJ018), which was supported by the Second Hospital of Jilin University.

\section{Footnote}

Reporting Checklist: The authors have completed the STROBE reporting checklist. Available at http://dx.doi. org/10.21037/apm-21-822

Data Sharing Statement: Available at http://dx.doi. org/10.21037/apm-21-822

Conflicts of Interest: All authors have completed the ICMJE uniform disclosure form (available at http://dx.doi. org/10.21037/apm-21-822). The authors have no conflicts of interest to declare.

Ethical Statement: The authors are accountable for all aspects of the work in ensuring that questions related to the accuracy or integrity of any part of the work are appropriately investigated and resolved. This study conformed to the tenets of the Declaration of Helsinki (as revised in 2013) and was approved by the Institutional Ethics Committee of the Second Hospital of Jilin University (2019-063). Because of the retrospective nature of the study, the requirement for informed consent was waived.

Open Access Statement: This is an Open Access article distributed in accordance with the Creative Commons Attribution-NonCommercial-NoDerivs 4.0 International License (CC BY-NC-ND 4.0), which permits the noncommercial replication and distribution of the article with the strict proviso that no changes or edits are made and the original work is properly cited (including links to both the formal publication through the relevant DOI and the license). See: https://creativecommons.org/licenses/by-nc-nd/4.0/.

\section{References}

1. Ma LY, Chen WW, Gao RL, et al. China cardiovascular diseases report 2018: an updated summary. J Geriatr Cardiol 2020;17:1-8.

2. Guan T, Ma J, Li M, et al. Rapid transitions in the epidemiology of stroke and its risk factors in China from 2002 to 2013. Neurology 2017;89:53-61.

3. Słomka A, Świtońska M, Sinkiewicz W, et al. Assessing Circulating Factor VIIa-Antithrombin Complexes in Acute Ischemic Stroke: A Pilot Study. Clin Appl Thromb Hemost 2017;23:351-9.

4. Feigin VL, Forouzanfar MH, Krishnamurthi R, et al. Global and regional burden of stroke during 1990-2010: findings from the Global Burden of Disease Study 2010. Lancet 2014;383:245-54.

5. Wang W, Jiang B, Sun H, et al. Prevalence, Incidence, and Mortality of Stroke in China: Results from a Nationwide Population-Based Survey of 480687 Adults. Circulation 2017;135:759-71.

6. Correll CU, Solmi M, Veronese N, et al. Prevalence, incidence and mortality from cardiovascular disease in patients with pooled and specific severe mental illness: a large-scale meta-analysis of 3,211,768 patients and 113,383,368 controls. World Psychiatry 2017;16:163-80.

7. Benjamin EJ, Blaha MJ, Chiuve SE, et al. Heart Disease and Stroke Statistics-2017 Update: A Report From the American Heart Association. Circulation 2017;135:e146-e603.

8. Chen WW, Gao RL, Liu LS, et al. China cardiovascular diseases report 2015: a summary. J Geriatr Cardiol 2017;14:1-10.

9. Redfern RE, Fleming K, March RL, et al. Thrombelastography-Directed Transfusion in Cardiac Surgery: Impact on Postoperative Outcomes. Ann Thorac Surg 2019;107:1313-8.

10. Martin G, Shah D, Elson N, et al. Relationship of Coagulopathy and Platelet Dysfunction to Transfusion Needs After Traumatic Brain Injury. Neurocrit Care 2018;28:330-7.

11. Yao X, Dong Q, Song Y, et al. Thrombelastography maximal clot strength could predict one-year functional outcome in patients with ischemic stroke. Cerebrovasc Dis 2014;38:182-90.

12. Jiang ZY, Ma JF, Wang Q, et al. A Systematic Review of the Value of Thrombelastography or Thromboelastometry for Prediction of Venous Thromboembolism. Zhongguo Shi Yan Xue Ye Xue Za Zhi 2017;25:1498-503. 
13. Corliss BM, Polifka AJ, Harris NS, et al. Laboratory assessments of therapeutic platelet inhibition in endovascular neurosurgery: comparing results of the VerifyNow P2Y12 assay to thromboelastography with platelet mapping. J Neurosurg 2018;129:1160-5.

14. Luz JRDD, Silva do Nascimento TE, Fernandes de Morais LV, et al. Thrombin Inhibition: Preliminary Assessment of the Anticoagulant Potential of Turnera subulata (Passifloraceae). J Med Food 2019;22:384-92.

15. Kayapinar O, Ozde C, Kaya A. Relationship Between the Reciprocal Change in Inflammation-Related Biomarkers (Fibrinogen-to-Albumin and hsCRP-to-Albumin Ratios) and the Presence and Severity of Coronary Slow Flow. Clin Appl Thromb Hemost 2019;25:1076029619835383.

16. van Rooy MJ, Duim W, Ehlers R, et al. Platelet hyperactivity and fibrin clot structure in transient ischemic attack individuals in the presence of metabolic syndrome: a microscopy and thromboelastography study. Cardiovasc Diabetol 2015;14:86.

17. Ganter MT, Hofer CK. Coagulation monitoring: current techniques and clinical use of viscoelastic point-of-care coagulation devices. Anesth Analg 2008;106:1366-75.

18. Kawano-Castillo J, Ward E, Elliott A, et al. Thrombelastography detects possible coagulation disturbance in patients with intracerebral hemorrhage with hematoma enlargement. Stroke 2014;45:683-8.

19. Connelly CR, Van PY, Hart KD, et al.

Thrombelastography-Based Dosing of Enoxaparin for Thromboprophylaxis in Trauma and Surgical Patients: A Randomized Clinical Trial. JAMA Surg 2016;151:e162069.

20. Dias JD, Norem K, Doorneweerd DD, et al. Use of Thromboelastography (TEG) for Detection of New Oral Anticoagulants. Arch Pathol Lab Med 2015;139:665-73.

21. Bowry R, Fraser S, Archeval-Lao JM, et al. Thrombelastography detects the anticoagulant effect of rivaroxaban in patients with stroke. Stroke 2014;45:880-3.

22. Shi Z, Zheng WC, Fu XL, et al. Hypercoagulation on Thromboelastography Predicts Early Neurological Deterioration in Patients with Acute Ischemic Stroke. Cerebrovasc Dis 2018;46:125-31.

23. Levy JH, Szlam F, Tanaka KA, et al. Fibrinogen and hemostasis: a primary hemostatic target for the management of acquired bleeding. Anesth Analg 2012;114:261-74.

24. Nielsen VG, Gurley WQ, Burch TM. The impact of factor XIII on coagulation kinetics and clot strength determined by thrombelastography. Anesth Analg 2004;99:120-3.
25. Feinbloom D, Bauer KA. Assessment of hemostatic risk factors in predicting arterial thrombotic events. Arterioscler Thromb Vasc Biol 2005;25:2043-53.

26. McCrath DJ, Cerboni E, Frumento RJ, et al. Thromboelastography maximum amplitude predicts postoperative thrombotic complications including myocardial infarction. Anesth Analg 2005;100:1576-83.

27. Kreutz RP, Owens J, Breall JA, et al. C-reactive protein and fibrin clot strength measured by thrombelastography after coronary stenting. Blood Coagul Fibrinolysis 2013;24:321-6.

28. Gurbel PA, Bliden KP, Guyer K, et al. Platelet reactivity in patients and recurrent events post-stenting: results of the PREPARE POST-STENTING Study. J Am Coll Cardiol 2005;46:1820-6.

29. Kreutz RP, Schmeisser G, Maatman B, et al. Fibrin clot strength measured by thrombelastography and outcomes after percutaneous coronary intervention. Thromb Haemost 2017;117:426-8.

30. Handtke S, Steil L, Palankar R, et al. Role of Platelet Size Revisited-Function and Protein Composition of Large and Small Platelets. Thromb Haemost 2019;119:407-20.

31. Arachchillage DRJ, Laffan M. Can Mean Platelet Volume Be Used as a Predictor of Vascular Disease? Problems and Pitfalls. Semin Thromb Hemost 2017;43:599-608.

32. Zhang P, Liu Y, Li J, et al. Cell proliferation in ependymal/ subventricular zone and nNOS expression following focal cerebral ischemia in adult rats. Neurol Res 2006;28:91-6.

33. Kovács S, Csiki Z, Zsóri KS, et al. Characteristics of platelet count and size and diagnostic accuracy of mean platelet volume in patients with venous thromboembolism. A systematic review and meta-analysis. Platelets 2019;30:139-47.

34. Hudzik B, Korzonek-Szlacheta I, Szkodziński J, et al. Association between multimorbidity and mean platelet volume in diabetic patients with acute myocardial infarction. Acta Diabetol 2018;55:175-83.

35. Tokuda N, Koga M, Ohara T, et al. Urgent Detection of Acute Type A Aortic Dissection in Hyperacute Ischemic Stroke or Transient Ischemic Attack. J Stroke Cerebrovasc Dis 2018;27:2112-7.

36. Zhang D, Li F, Du X, et al. Diagnostic accuracy of biomarker D-dimer in patients after stroke suspected from venous thromboembolism: A diagnostic meta-analysis. Clin Biochem 2019;63:126-34.

37. You LR, Tang M. The association of high D-dimer level with high risk of ischemic stroke in nonvalvular atrial fibrillation patients: A retrospective study. Medicine 
(Baltimore) 2018;97:e12622.

38. Farm M, Siddiqui AJ, Onelöv L, et al. Age-adjusted D-dimer cut-off leads to more efficient diagnosis of venous thromboembolism in the emergency department: a comparison of four assays. J Thromb Haemost 2018;16:866-75.

(English Language Editor: C. Betlazar-Maseh)

Cite this article as: Zhao F, Yu P, Lv X, Pang M, Zhao L, Zhang X, Zhao L. Value of thromboelastography combined with anticoagulant detection in patients over 60 years of age with cardiovascular or cerebrovascular disease. Ann Palliat Med 2021;10(5):5407-5416. doi: 10.21037/apm-21-822 